\title{
On the Parity Problem in One-Dimensional Cellular Automata
}

\author{
Heather Betel \\ School of Electrical Engineering and Computer Science \\ University of Ottawa \\ Ottawa, Canada \\ hbetel@site.uottawa.ca
}

\author{
Pedro P.B. de Oliveira \\ Universidade Presbiteriana Mackenzie \\ Faculdade de Computação e Informática \\ São Paulo, Brazil \\ pedrob@mackenzie.br
}

\author{
Paola Flocchini \\ School of Electrical Engineering and Computer Science \\ University of Ottawa \\ Ottawa, Canada \\ flocchin@site.uottawa.ca
}

\begin{abstract}
We consider the parity problem in one-dimensional, binary, circular cellular automata: if the initial configuration contains an odd number of $1 \mathrm{~s}$, the lattice should converge to all 1s; otherwise, it should converge to all 0s. It is easy to see that the problem is ill-defined for even-sized lattices (which, by definition, would never be able to converge to 1 ). We then consider only odd lattices.

We are interested in determining the minimal neighbourhood that allows the problem to be solvable for any initial configuration. On the one hand, we show that radius 2 is not sufficient, proving that there exists no radius 2 rule that can possibly solve the parity problem from arbitrary initial configurations. On the other hand, we design a radius 4 rule that converges correctly for any initial configuration and we formally prove its correctness. Whether or not there exists a radius 3 rule that solves the parity problem remains an open problem.
\end{abstract}

\section{Introduction}

Understanding the nature of computations within cellular automata remains an elusive problem. In fact, in spite of their long-proclaimed ability to perform computations, very little is still known as to how we should design the local state transitions towards achieving a given global behaviour. As examples are designed or found by search, it is inevitable to try to understand their underlying programming language; but the truth is, to this date, every attempt along these lines has fallen into the strenuous effort of trying to tame local state patterns towards the global state target, or trying to make sense of the latter in terms of the former [6].

On the other hand, studying how to employ local actions to achieve desirable global behaviours is of utmost importance and extensively investigated in many other evolving systems (e.g., distributed systems, mobile robots, population protocols). In such systems, in fact, understanding the limitations and the power of local interactions to solve global computations has immediate implications on the design of efficient and scalable solutions (e.g., see [1, 2, 10, 13]). Cellular automata (CAs) are the simplest possible evolving systems, and understanding the impact that neighbourhood size has on computability could have consequences for more complex systems based on local interactions.

This paper is aligned with these efforts. Here, we concentrate on the one-dimensional parity problem, which has essentially the objective of figuring out the parity of an arbitrary binary string, by means of a one-dimensional, binary cellular automaton [14]. The parity problem is a well-known benchmark 
task in various areas of computer science, typically camouflaged under the XOR operation on a binary input, as in artificial neural networks [7], but it also lends itself to the context of cellular automata, as a typical case of a global problem that has to be solved by purely local processing. The problem is formulated under periodic boundary conditions and arbitrary finite lattice size, so that, if the parity of the global configuration is odd, the lattice is supposed to lead to an homogeneous configuration with only $1 \mathrm{~s}$; otherwise, it should converge to all 0s [9].

The notion of parity has appeared quite often in the CA literature, even if implicitly, as it bears relevance to the related notion of additivity of CA rules [3, 15]. However, the parity problem per se has not been extensively studied, particularly in comparison with the well-known benchmark CA task of density classification, where the aim is to determine the most frequent bit in the initial configuration of an odd-sized lattice, also by reaching an homogeneous configuration. The density classification problem, in fact, has been extensively investigated and fully understood in odd-sized lattices. In particular, it has been shown that there exists no single rule able to solve the problem for any arbitrary initial configuration. Combinations of rules have been devised, however, as well as probabilistic solutions to the problem (e.g., see [12, 4, 5, 16]).

An advantage in favour of the parity problem is that, from the perspective of automata theory, it is simpler than its kin, insofar as the notion of parity can be handled by finite automata, whereas the ability to compare arbitrarily variable quantities (which is inherent to density classification) requires at least a pushdown automaton [8]. In fact, the increased simplicity of the parity problem is reflected in the fact that it is easier to find good rules for it, by searching, than for density classification [16]. Therefore, there are strong reasons for considering the parity problem generally more tractable and amenable to analysis, which makes it a serious candidate for case studies that might help the understanding of the nature of computation in CAs in general.

The parity problem is ill-defined for even-sized lattices (by definition, an all 1 configuration converges to an all 0 configuration making it impossible for any rule to converge to 1). Modifying the definition of the problem to allow the target homogeneous configuration to be achieved only once, and not as a fixed point, the problem becomes solvable also for even lattices. In fact, by relying on this variation, it can be perfectly solved by a carefully engineered sequence of rule applications, quite surprisingly, of elementary cellular automata [11]. However, if we do not want to change the definition of the problem, it is then necessary to restrict the study to odd-sized lattices. We then say that a CA rule is perfect if it solves the parity problem for arbitrary initial odd-sized configurations.

Unlike the density classification problem, we show that the parity problem can, indeed, be solved by a single rule. Besides being interested in its general solvability, we are also interested in determining the minimal neighbourhood that allows the construction of a perfect rule. With this goal in mind, we first prove that radius 2 is not sufficient for a perfect rule to exist. We first identify several constraints to which such a perfect rule is subject and we show that no rule is feasible with all of these constraints. We then show that the problem becomes solvable when CAs have radius 4: our proof is constructive as we design a perfect rule and we prove its correctness. We leave open the case of radius 3 , for which there is strong empirical evidence that no perfect rule exists, but that there might be radius 3 rules that would solve the problem for prime-sized lattices [16].

\section{Notation and Basic Facts}

We consider one-dimensional, binary cellular automata (CAs) on finite lattices with periodic boundary conditions. Let $f:\{0,1\}^{2 r+1} \rightarrow\{0,1\}$ denote the local rule of a CA with radius $r$. The global dy- 
namics of a one-dimensional cellular automaton composed of $n$ cells and radius $r$ is then defined by the global rule (or transition function): $F:\{0,1\}^{n} \rightarrow\{0,1\}^{n}$ s.t. $\forall \mathbf{X} \in\{0,1\}^{n}, \forall i \in\{0, \ldots, n-1\}, F(X)_{i}=$ $f\left(x_{i-r} \ldots, x_{i}, \ldots, x_{i+r}\right)$, where all operations on indices are modulo $n$.

A fixed point $\mathbf{P} \in\{0,1\}^{n}$ of a circular CA with global transition rule $F$ is a configuration $\mathbf{P}$ such that $F(\mathbf{P})=\mathbf{P}$.

We say that a cellular automaton converges to a configuration $P$ from configuration $X^{0}$ if $P$ is a fixed point and if for some finite $n, F^{n}\left(X^{0}\right)=P$ where $F^{n}$ is the $n^{\text {th }}$ iteration of $F$. We are particularly interested in the homogeneous configurations as fixed points and will refer to these as the 0 -configuration and the 1-configuration.

We say that a local rule solves the parity problem if, starting from an arbitrary initial configuration, on an arbitrarily sized lattice, the cellular automaton converges to the 0-configuration, if and only if the initial configuration contains an even number of $1 \mathrm{~s}$, and converges to the 1-configuration otherwise.

Since a rule solving the parity problem must converge to the homogeneous configurations, we have our first two properties, of perfect rules.

Property 1 If $f$ solves the parity problem, then $f(0, \cdots, 0)=0$ and $f(1, \cdots, 1)=1$.

It is immediately obvious that, by definition, no solution exists for even-sized lattices.

Theorem 1 Consider circular CAs with radius $r$ and even size $n$. There exists no rule that works correctly from any initial configuration.

PROOF Trivially $f(1 \ldots 1)=0$ otherwise the configuration with all 1 s would incorrectly converge to 1 . Since $(11111 \ldots 111)$ is not a fixed point, it follows that no initial configuration can ever converge to the 1-configuration.

For this reason, from now on, we consider only odd-sized lattices and we call a rule perfect if it solves the parity problem for any odd-sized lattice, starting from any initial configuration.

We now recall the definition of de Bruijn graphs, which are useful tools for representing CA rules and which will be used in the subsequent sections. The de Bruijn graph of a local rule of radius $r$ is a directed graph on $2^{2 r}$ nodes, one for each value in the set $\{0,1\}^{2 r}$. There is an edge from node $x_{0} \ldots x_{2 r-1}$ to node $y_{1} \ldots y_{2 r}$ if $x_{i}=y_{i}$ for all $i$ from 1 to $2 r-1$. These edges are labelled with the value of the local function at $\left(x_{0}, \ldots, x_{2 r-1}, y_{2 r}\right), f\left(x_{0}, \ldots, x_{2 r-1}, y_{2 r}\right)=f\left(x_{0}, y_{1}, \ldots, y_{2 r}\right)$. Note that the shape of the de Bruijn graph for a local rule of a given family (i.e., those with the same neighbourhood and states) is fixed, only the edge labels change. For example, Figure 1 shows the de Bruijn graph for a radius 1 rule.

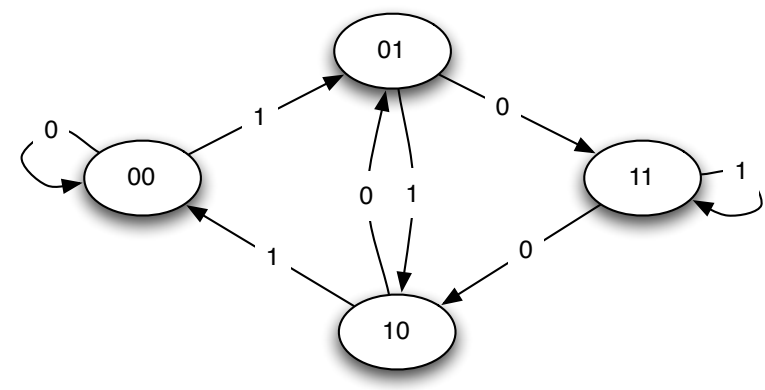

Figure 1: De Bruijn graph for the local parity rule (150).

The following is a necessary condition for parity preservation. 
Property 2 A rule preserves the parity of a configuration if active transitions always come in pairs. That is, given a local rule $f$ of radius $r$, and any configuration $\left(x_{0}, \ldots, x_{n-1}\right)$, the number of times that $f\left(x_{i-r}, \ldots, x_{i}, \ldots, x_{i+r}\right) \neq x_{i}$ is even.

It is also very simple to see that no solution exists for elementary circular CAs (i.e., with radius 1).

Theorem 2 There exists no perfect rule for elementary CAs.

PROOF From Property 1, for any perfect rule, we must have $f(000)=0$ and $f(111)=1$. Now consider a configuration containing a single 1 . In order to both maintain parity and move towards convergence, we must have $f(100)=f(010)=f(001)=1$. Similarly, from the singleton 0 , we must have $f(110)=f(101)=f(011)=0$. So the only possible perfect rule is the local parity check (rule 150). However, it is easy to see that such a rule does not converge from several initial configurations, for example from $(\ldots 0001000 \ldots)$.

\section{Impossibility with Radius 2}

In this section we show that with radius 2 it is impossible to construct a perfect parity rule.

Our aim is to show, first, several necessary transitions for a perfect rule, and second, the existence of a limited set of feasible pre-images for the two final homogeneous configurations. Each possible pair of feasible pre-images further induces necessary transitions for a perfect rule, significantly reducing the space of possible perfect rules. We conclude the proof by running exhaustive tests to verify that in this set there exists no perfect rule having the necessary transitions. We begin with a series of lemmas that force certain transitions to 0 or 1 .

Consider the de Bruijn graph for radius 2 rules. A pre-image of the final 0-configuration corresponds to a cycle of odd size and even parity. A pre-image of the final 1-configuration is a cycle of odd size and odd parity. Let $\mathscr{B}_{0}$ be the subgraph containing only the edges corresponding to transitions to 0 and $\mathscr{B}_{1}$ the subgraph containing the edges corresponding to transitions to 1.

Lemma 1 In a perfect rule, three or five of the following must transition to 1: (10000), (01000), (00100), (00010), (00001).

PROOF A configuration consisting of a single 1 must eventually converge to all $1 \mathrm{~s}$, hence the number of $1 \mathrm{~s}$ in the configuration must increase. Furthermore, in order to maintain parity, it must increase to an odd number. The five configurations above are the only ones occurring at the local level that are not all 0 , and therefore 3 or 5 of them must go to 1 .

Similarly,

Lemma 2 In a perfect parity rule, three or five of the following must transition to 0 :

(01111),(10111),(11011),(11101),(11110).

Lemma 3 Neither $\mathscr{B}_{0}$ nor $\mathscr{B}_{1}$ can contain a cycle of even length and odd parity.

PROOF A cycle of even length in either $\mathscr{B}_{0}$ or $\mathscr{B}_{1}$ will become a sequence having even parity at the next iteration since it will be either all 0 s or an even number of $1 \mathrm{~s}$, so this cycle itself will have changed parity. Assume that the de Bruijn graph of a rule $F$ admits such a cycle, let $C$ be such a cycle and let $P$ be a cycle of odd length passing through a node of $C$. For the rule to be perfect, $F(P)$ must have the same parity as 
$P$. Now consider a new cycle $P^{\prime}$ formed from $P$ by adding the cycle $C$ where $P$ passes through it. Since $C$ has even length, $P^{\prime}$ has odd length. Since $C$ has odd parity, $P$ and $P^{\prime}$ have different parity. However, since $F(C)$ has even parity, $F\left(P^{\prime}\right)=F(P)$, hence the parity of $P^{\prime}$ has changed and $F$ cannot be perfect.

Lemma 4 In a perfect parity rule either: $i) f(10101)=1$ and $f(01010)=0$, or ii) $f(10101)=0$ and $f(01010)=1$.

PROOF This is a direct consequence of Lemma 3 since $f(10101)=f(01010)$ would imply the existence of the even cycle with odd parity $(1010,0101,1010,0101,1010,0101)$ either in $\mathscr{B}_{0}$ or in $\mathscr{B}_{1}$.

Lemma 5 In a perfect parity rule, it is impossible to have four or more consecutive Os in a pre-image of the 0-configuration.

PROOF Let the pattern $(0000)$ be present in a pre-image $P$ of the 0 -configuration. Then the following neighbourhood configurations must also be present and must be transitioning to 0: (10000) and (00001). By Lemma 1, we must then have the following configurations transitioning to 1, so they may not occur in P: (01000), (00100), (00010). Hence our group of four 0s must be both preceded and followed by at least two 1s, thus entailing that we have the necessary transition set $S=$ $\{f(11000), f(00011), f(10000), f(00001)\} \rightarrow 0$. Consider now an initial configuration containing a single 1 surrounded by 0 s. From $S$, we have that 0001000 can only grow to 0011100 , but, again from $S$, we have that, from 0011100 , no growth is possible anymore, which is a contradiction.

Analogously, we have:

Lemma 6 In a perfect parity rule, it is impossible to have four or more consecutive 1 s in a pre-image of the 1-configuration.

From Lemma 3, any feasible pre-image of the 0-configuration (resp. 1-configuration) is either a simple odd cycle $c$ with even (resp. odd) parity, or the composition of cycles not containing any even cycle of odd parity.

So, to identify feasible pre-images for final configurations for lattice size $n$ in the de Bruijn graph, we have to find at least one cycle of size $n$ to be labeled 0 and one to be labeled 1, having the property that they do not include:

(i) the self-loops (which are forbidden by Lemmas 5 and 6

(ii) the 2-cycle $(0101,1010)$ (which is forbidden by Lemma 4); and

(iii) an even cycle with odd parity (Lemma 3).

By inspecting all cycles of size 5, we obtain that:

Lemma 7 In a perfect rule at least one of these three cycles in the de Bruijn graph must transition to 1: $B_{1}^{5}=(0011,0111,1110,1100,1001)$ (corresponding to configuration: 00111)

$B_{2}^{5}=(0000,0001,0010,0100,1000)$ (corresponding to configuration: 00001)

$B_{3}^{5}=(0101,1011,0110,1101,1010)$ (corresponding to configuration: 01011)

and one of these must transition to 0 :

$W_{1}^{5}=(0001,0011,0110,1100,1000)$ (corresponding to configuration: 00011)

$W_{2}^{5}=(0111,1111,1110,1101,1011)$ (corresponding to configuration: 01111)

$W_{3}^{5}=(0010,0101,1010,0100,1001)$ (corresponding to configuration: 00101) 
PROOF $B_{1}^{5}, B_{2}^{5}$ and $B_{3}^{5}$ (resp. $W_{1}^{5}, W_{2}^{5}$ and $W_{3}^{5}$ ) are the only cycles corresponding to feasible pre-images for the 1-configuration (resp. 0-configuration) for lattices of size 5, which do not violate Lemmas 4, 5. and 6 .

Consider, now, lattices of size 7. All cycles of length 7 have been enumerated and the only cycles that do not contradict Lemmas 3, 4, 5, and 6 and correspond to feasible pre-images of the 1-configuration are:

$B_{1}^{7}=(0000,0001,0011,0111,1110,1100,1000)$ (configuration: 0000111)

$B_{2}^{7}=(0001,0011,0110,1101,1010,0100,1000)$ (configuration: 0001101)

$B_{3}^{7}=(0001,0010,0101,1011,0110,1100,1000)$ (configuration: 0001011)

$B_{4}^{7}=(1001,0011,0110,1100,1001,0010,0100)$ (configuration: 1001100)

Analogously, the only cycles which do not violate Lemmas 3, 4, 5, and 6 and correspond to feasible pre-images of the 0-configuration are:

$W_{1}^{7}=(0001,0011,0111,1111,1110,1100,1000)$ (configuration: 0001111)

$W_{2}^{7}=(0010,0101,1011,0111,1110,1100,1001)$ (configuration: 0010111)

$W_{3}^{7}=(0011,0111,1110,1101,1010,0100,1001)$ (configuration: 0011101)

$W_{4}^{7}=(0110,1100,1001,0011,0110,1101,1011)$ (configuration: 0110011)

From simple observation, we can rule out some of these cycles and combinations of cycles.

Lemma 8 A perfect rule of radius 2 cannot have $W_{1}^{5}$ as a pre-image of the 0 -configuration.

Proof Cycle $W_{1}^{5}$ shares at least one transition in common with each of the possible pre-images of the 1-configuration of size 7. For example, $W_{1}^{5}, B_{1}^{7}$ and $B_{2}^{7}$ all share the edge $(0001,0011)$ in the de Bruijn graph. Cycles $W_{1}^{5}$ and $B_{3}^{7}$ share $(0010,0101)$, and $W_{1}^{5}$ shares $(0011,0110)$ with $B_{4}^{7}$.

Lemma 9 A perfect rule of radius 2 cannot have $B_{2}^{7}$ as a pre-image of the 1-configuration.

PROOF First, if $B_{2}^{7}$ is a pre-image of the 1-configuration, then $W_{2}^{5}$ is a pre-image of the 0-configuration of size 5 since $B_{2}^{7}$ and $W_{3}^{5}$ share $(1010,0100)$. Cycle $B_{2}^{7}$ also has transitions in common with $W_{1}^{7}$, $W_{3}^{7}, W_{4}^{7}$. It has no common transitions with $W_{2}^{7}$, however, $W_{2}^{7}$ and $W_{2}^{5}$ together form a cycle, namely, $(0111,1110,1101,1011)$, in violation of Lemma 3 .

Similarly, we can show,

Lemma 10 A perfect rule of radius 2 cannot have $B_{3}^{7}$ as a pre-image of the 1-configuration.

In fact, we can now restrict to very well defined possible cases.

Lemma 11 A perfect rule of radius 2 must have $W_{2}^{5}$ as a pre-image of the 0 -configuration, $B_{2}^{5}$ as a pre-image of the 1-configuration and either

- $B_{1}^{7}$ as a pre-image of the 1-configuration and $W_{4}^{7}$ as a pre-image of the 0 -configuration, or

- $B_{4}^{7}$ as a pre-image of the 1-configuration and $W_{1}^{7}$ as a pre-image of the 0-configuration. 
PROOF From the lemmas above, we know that the only possible pre-images of the 1-configuration of size 7 are $B_{1}^{7}$ and $B_{4}^{7}$. Cycle $B_{1}^{7}$ has transitions in common with all possible pre-images of the 0 -configuration except $W_{4}^{7}$. Of the 5-cycle pre-images of the 1-configuration, only $B_{2}^{5}$ is compatible with $W_{4}^{7}$. Of the possible pre-images of the 0 -configuration of size 5 , neither $W_{2}^{5}$ nor $W_{3}^{5}$ poses any conflict, however, $W_{3}^{5}$ makes it impossible to have any cycles of length 3 going to the 1-configuration, so that all configurations having a period of size 3 , (i.e. configurations of the form $(001001001 \cdots 001)$ will fail to converge). The proof is analogous beginning with cycle $B_{4}^{7}$.

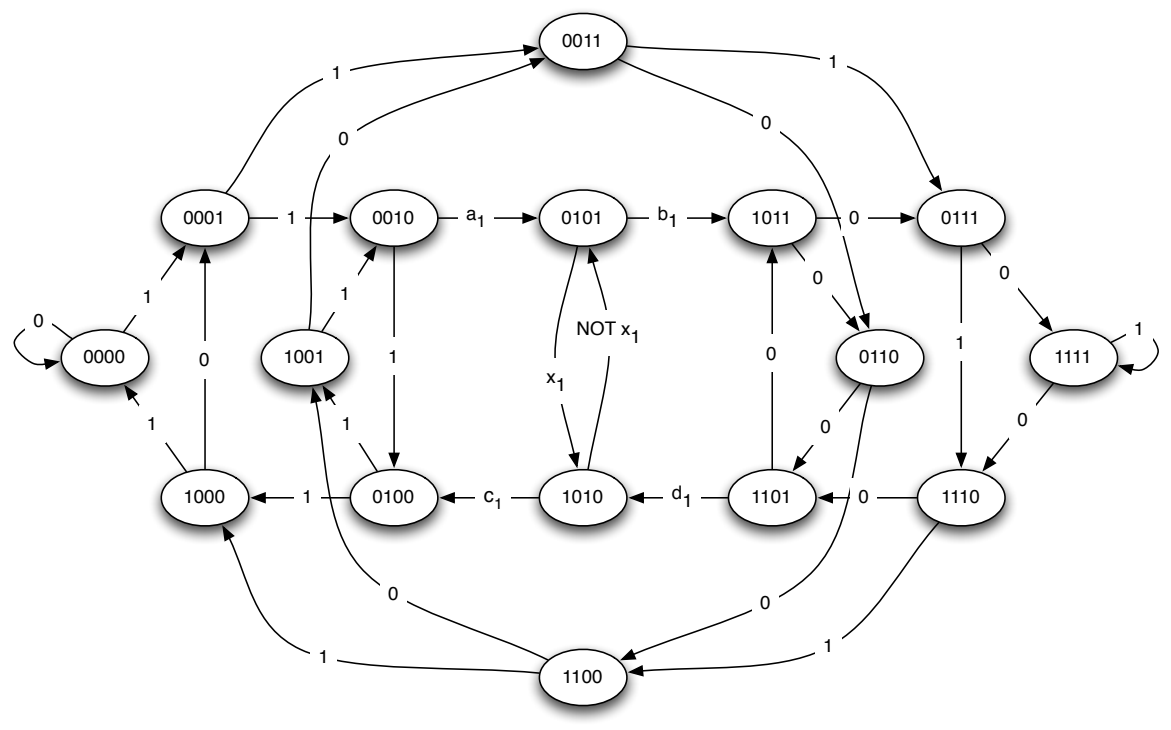

Figure 2: Possible perfect rule for radius 2, with $B_{1}^{7}$ and $W_{4}^{7}$.

We can finally conclude:

Theorem 3 There is no perfect parity rule of radius 2.

PROOF From the lemmas in this section it follows that for a perfect parity rule of radius 2, we must have one of the de Bruijn graphs shown in Figure 2 or Figure 3. Note that, in these graphs, depending on the choice of $x_{i}$, we can place further restrictions on the remaining unknown edges (again, due to the lemmas in this section). Consider Figure 2, if $x_{1}=1$, then in order to avoid creating cycles with an odd number of transitions, we must have $a_{1} \neq c_{1}, b_{1} \neq d_{1}$ from cycles $(0001,0010,0101,1010,0100,1000)$ and $(0101,1011,0111,1110,1101,1010)$. When $x_{1}=0$ by contrast, we must have $a_{1}=c_{1}, b_{1}=d_{1}$. The constraints on $a_{2}, b_{2}, c_{2}$, and $d_{2}$ resulting from the choice of $x_{2}$ are the same. Testing has shown that all 16 of the resulting rules will fail for some initial configurations.

A final note concerns lattices of prime size. It has been conjectured, in the case of radius 3 , that there may exist rules with the desired behaviour on arbitrary lattices of prime size [16]. While the case of radius 3 is still open, we can show that the impossibility result for radius 2 holds even if we restrict the discussion to prime-sized lattices.

Theorem 4 There is no radius 2 rule the always solves the parity problem even restricting to lattices of prime size. 


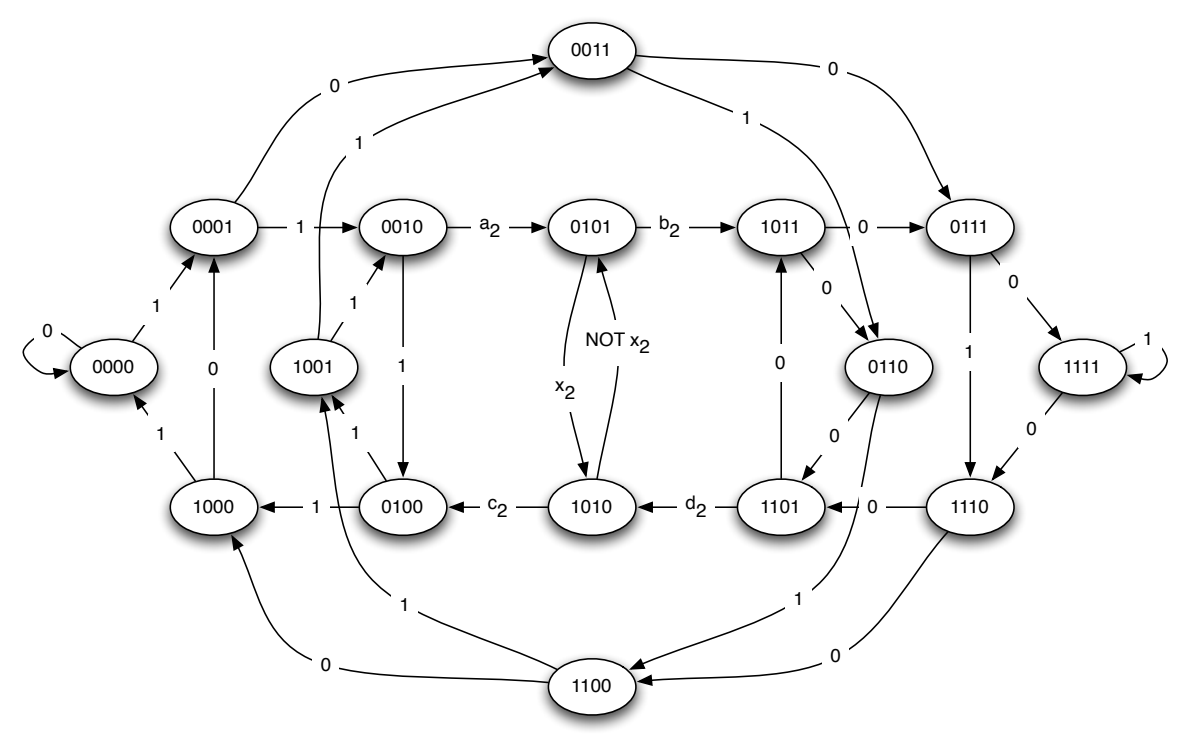

Figure 3: Possible perfect rule for radius 2, with $B_{4}^{7}$ and $W_{1}^{7}$.

PROOF Restricting to prime size lattices, we can no longer use Lemma 11. This introduces only a few possible extra cases using $B_{3}^{5}$ or $W_{3}^{5}$. Consider rules containing $B_{3}^{5}$. As before, we can eliminate $W_{1}^{5}$, $B_{2}^{7}$ and $B_{3}^{7}$ as pre-images of the 0 - and 1- configurations. In addition, $B_{3}^{5}$ conflicts with $W_{2}^{7}$ and $W_{3}^{7}$ on the edge from 0101 to 1011 and with $W_{4}^{7}$ on the edge from 0110 to 1101 . Hence, we must have $W_{1}^{7}$ as the 7-cycle pre-image of the 0-configuration. Now, $B_{1}^{7}$ conflicts with $W_{1}^{7}$, so we are left with $B_{4}^{7}$ as the 7-cycle pre-image of the 1-configuration. Since $B_{4}^{7}$ conflicts with $W_{3}^{5}$ on the edge from 0100 to 1001 , we are left with $W_{2}^{5}$ as the 5-cycle pre-image of the 0-configuration. These results are illustrated in the graph of Figure 4. Proposition 2 dictates that the edges labeled $a_{3}$ must be the same, as will be the edges labeled $b_{3}$. Now consider rules containing $W_{3}^{5}$. Similar analysis shows that must have $B_{2}^{5}, W_{4}^{7}$ and $B_{1}^{7}$, as illustrated in Figure 5. Testing has shown that all 8 of these rules will also fail for some initial configurations of prime length.

\section{A Perfect Rule with Radius 4}

We now describe the construction of a rule with radius 4 having the desired properties: parity preservation and convergence. The intention is to first give the reader an intuitive understanding of how the rule works and how it was developed. Formal proofs will follow in the subsequent section.

\subsection{Rule BFO}

The most compact representation of rule BFO that we propose for solving the parity problem in any lattice of odd size is given in Figure 6 and corresponds to rule number:

12766019579927887748828308783632125137208948629571434199404394002671695991869267727 -

072917454377539194754200976283425175983876539715064584172642413634846720 


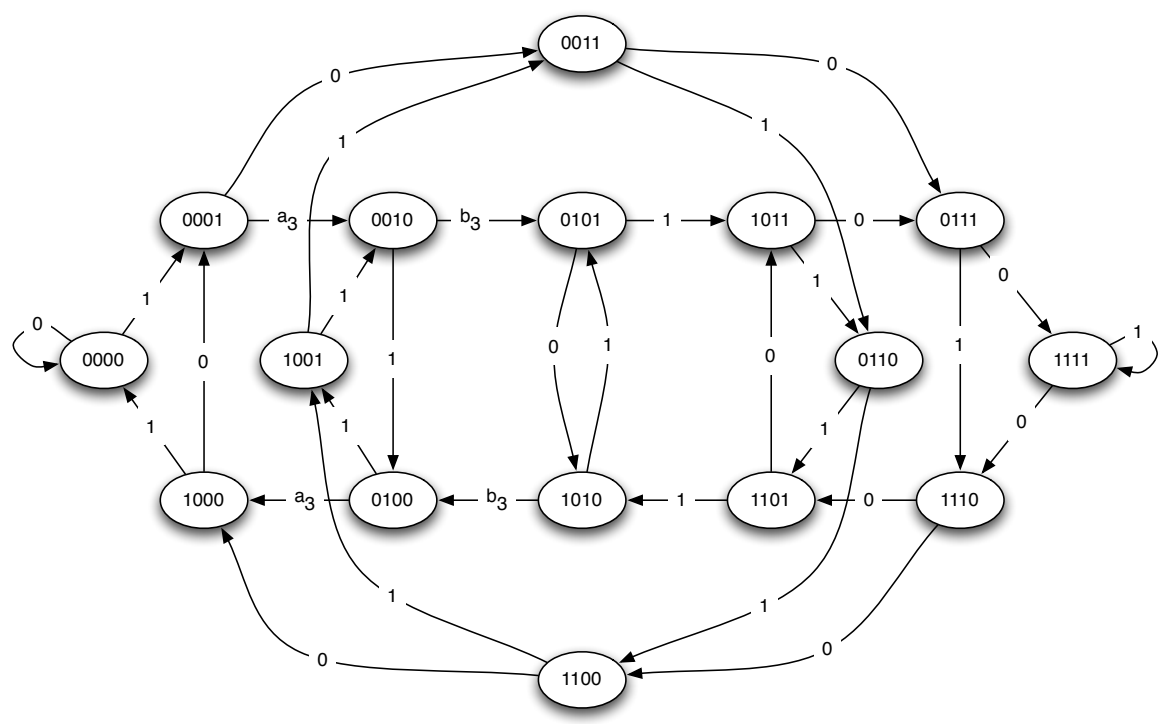

Figure 4: Additional possible perfect rules for radius 2 on prime lattices with $W_{2}^{5}$.

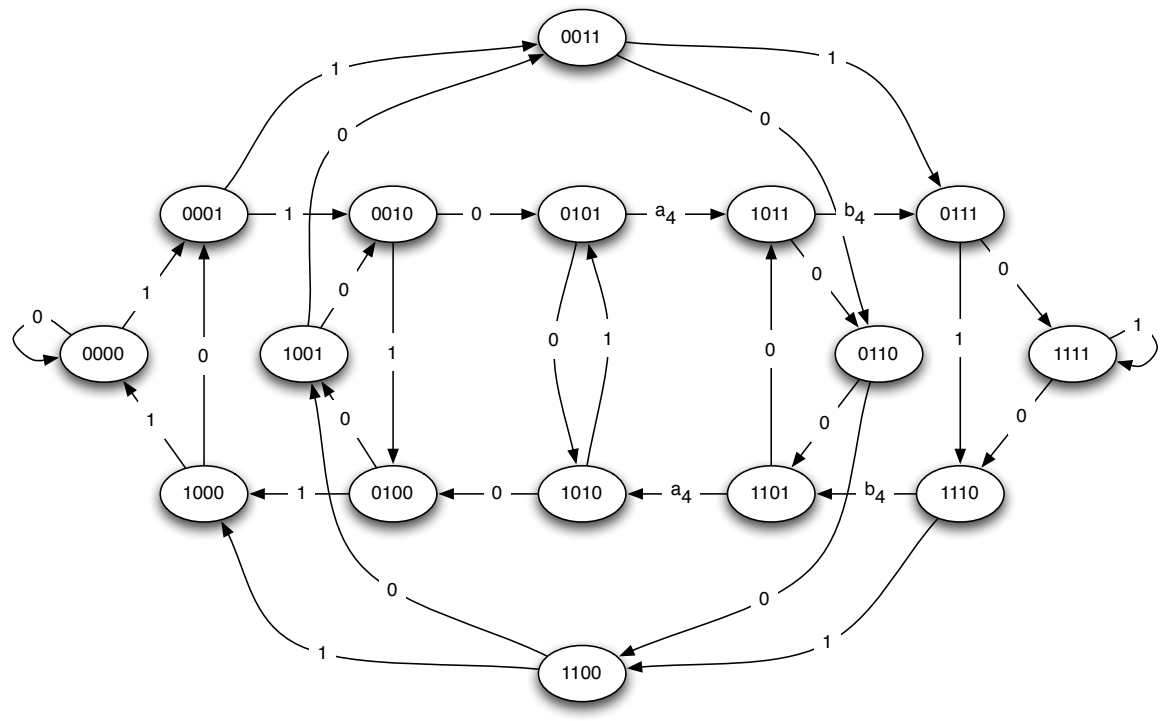

Figure 5: Additional possible perfect rules for radius 2 on prime lattices with $B_{2}^{5}$.

in Wolfram's lexicographic ordering scheme. The figure shows all active transitions (i.e., transitions that change the current state). However, it is often easier to explain why and how the rule works using a less compact form, where pairs of rules can be made explicit. This form of the rule is given in Figure 7 , we will be referring to this representation in the remainder of this section.

We now describe the intended behaviour of the rule before proving its correctness. Consider an initial configuration $X^{0}$ as being formed by blocks $b_{i}$ of consecutive 1s separated by blocks $w_{i}$ of consecutive 0s: $X^{0}=\left(b_{1}^{0}, w_{1}^{0}, b_{2}^{0}, w_{2}^{0}, \cdots, b_{k}^{0}, w_{k}^{0} \cdots\right)$. The idea of our construction is to have a block $b_{i}$ of 1 s propagate 


\begin{tabular}{|c|c|}
\hline $\begin{array}{c}\text { Neighbourhood } \\
\text { configurations }\end{array}$ & $\begin{array}{c}\text { Output } \\
\text { bit }\end{array}$ \\
\hline$* 11100 * * *$ & 1 \\
$11100 * * * *$ & 1 \\
$* 00100 * * *$ & 1 \\
$00100 * * * *$ & 1 \\
$* * 01 \mathbf{0 1 0 0 *}$ & 1 \\
\hline $11101 * * * *$ & 0 \\
$* 0101 * 0 * *$ & 0 \\
$* * 01 \mathbf{1 0} * * *$ & 0 \\
$* * * 1 \mathbf{1 0 1 1 0}$ & 0 \\
$* * * 0110 * *$ & 0 \\
$* * * * \mathbf{1 1 0 1 *}$ & 0 \\
\hline
\end{tabular}

Figure 6: Minimised rule BFO (the asterisk $*$ refers to any value).

\begin{tabular}{|c|c|c|c|c|}
\hline Name & $\begin{array}{l}\text { Neighbourhood } \\
\text { configurations }\end{array}$ & $\begin{array}{c}\text { Output } \\
\text { bit }\end{array}$ & & \\
\hline$T_{1}:$ & $* 11100 * * *$ & 1 & & \\
\hline$T_{2}:$ & $11100 * * * *$ & 1 & Pair & Behaviour \\
\hline$T_{3}:$ & $* 00100 * * *$ & 1 & $T_{1}, T_{2}$ & Rightward growth of 1-blocks \\
\hline$T_{4}:$ & $00100 * * * *$ & 1 & $T_{3}, T_{4}$ & Rightward growth of 1-blocks \\
\hline$T_{7}:$ & $* * 010100 *$ & 1 & $T_{5}, T_{6}$ & Annihilation of 11 \\
\hline$T_{5}:$ & $* * * 0110 * *$ & 0 & $T_{7}, T_{8}$ & Local shift \\
\hline$T_{6}:$ & $* * 0110 * * *$ & 0 & $T_{9}, T_{10}$ & Leftward growth of 0-blocks \\
\hline$T_{8}:$ & $* 010100 * *$ & 0 & $T_{9}, T_{11}$ & Start of 0-growth \\
\hline$T_{9}:$ & $* * * 11101 *$ & 0 & $T_{9}, T_{12}$ & Local adjustment \\
\hline$T_{10}:$ & $111010 * * *$ & 0 & & \\
\hline$T_{11}:$ & $1110111 * *$ & 0 & & \\
\hline$T_{12}:$ & $* * 1110110$ & 0 & & \\
\hline
\end{tabular}

Figure 7: Active rule transitions (left) and behaviour of combinations of rules (right).

to the right, two cells per iteration, until a stopping condition or convergence has been reached. Such propagation might result in merging the block with the next $b_{i+1}$ (if the corresponding $w_{i}$ is of even size). When the merger does not occur (because $\left|w_{i}\right|$ is odd or due to some other condition), there will be a propagation of $0 \mathrm{~s}$ to the left, led by a block of the form (01). Such counter-propagation might result in the total annihilation of the block of 1s. Otherwise, it will result in the creation of a single 1 surrounded by 0 s, which will start propagating to the right again. We will show that such behaviour reduces the number of blocks, eventually converging to an homogenous configuration.

We now describe some properties of the rule that can easily be derived by construction and that give an intuition for the reasons for the behaviour described above. Note that, by construction, the rule's transitions always occur in pairs; in other words, whenever a transition occurs in a cell, another transition occurs in its neighbourhood. It is useful to describe the behaviour of each pair and we will also use these pairs to prove that parity is being preserved.

- Rightward growth of 1-blocks. A singleton 1 grows to the right, by two 1 s at each step, if it is 
preceded and followed by at least two $0 \mathrm{~s}$, as prescribed by transitions $T_{3}$ and $T_{4}$. A block of three or more $1 \mathrm{~s}$ grows to the right if it is followed (on the right) by at least two 0s. This behaviour is created by the pair of transitions $T_{1}$ and $T_{2}$.

- Annihilation of pairs of $1 \mathrm{~s}$. As a consequence of transitions $T_{5}$ and $T_{6}$, an isolated pair of $1 \mathrm{~s}$ is always eliminated.

— Leftward growth of 0-blocks. A (01) block moves to the left, leading a growing block of 0s (at a growth rate of two 0s per step) if there are at least three 1s to its left and one of the following: $(i)$ at least three $1 \mathrm{~s}$ to the right of the 0 (the growth is obtained by the pair of transitions, $\left(T_{9}, T_{11}\right)$; or $(i i)$ at least one 0 to its right (due to $T_{9}$ and $\left.T_{10}\right)$. Note that the pair $\left(T_{9}, T_{11}\right)$ starts the growth of a 0-block, while the pair $\left(T_{9}, T_{10}\right)$ continues the growth as far as possible.

- Local Shift. A (101) block is transformed into (110) if there are a 0 on its left and at least two 0s on its right (combination of transitions $T_{7}$ and $T_{8}$ ).

— Local Adjustment. Finally, if a (0110) block is preceded by at least three 1s that is, (..1110110 ...) occurs, in order to avoid parity errors due to the annihilation of the pair of $1 \mathrm{~s}$, we force the creation of a solid block of $0 \mathrm{~s}$ to the right of the existing block of $1 \mathrm{~s}$ with transition pair $\left(T_{9}, T_{12}\right)$, so that $(\ldots 1110110 \ldots)$ becomes $(\ldots 1000000 \ldots)$.

Examples of the evolution of the rule are given in Figure 8 .
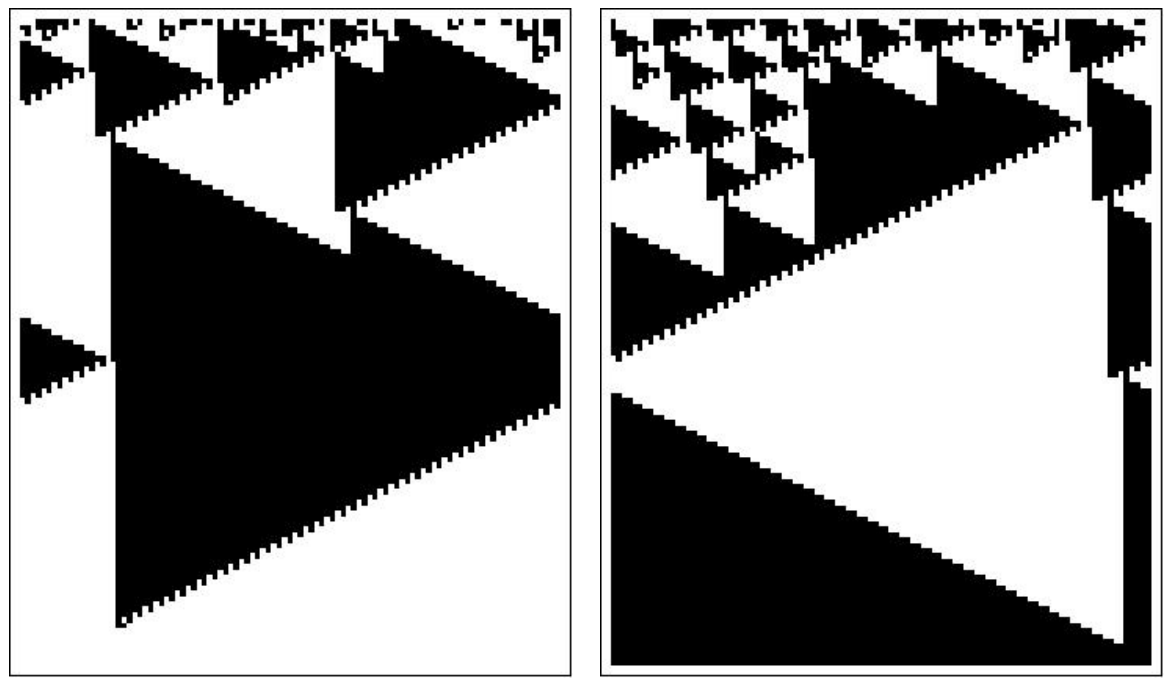

Figure 8: Evolution of rule BFO for even parity (left) and odd parity (right). A black cell corresponds to 1 , a white cell corresponds to 0 . The initial configuration is at the top and time goes downward.

\subsection{Correctness}

In order to show that the rule we have constructed (or in fact any rule) performs a perfect parity check, we must prove that it preserves parity at every iteration, and that it always converges in finite time to an homogeneous configuration. We begin with the proof of parity conservation.

\subsubsection{Parity Preservation}

A rule preserves the parity of a configuration if active transitions always come in pairs. That is, given a local rule $f$ of radius $r$, and any configuration $\left(x_{0}, \ldots, x_{n-1}\right)$, the number of times that $f\left(x_{i-r}, \ldots, x_{i}, \ldots, x_{i+r}\right) \neq$ 


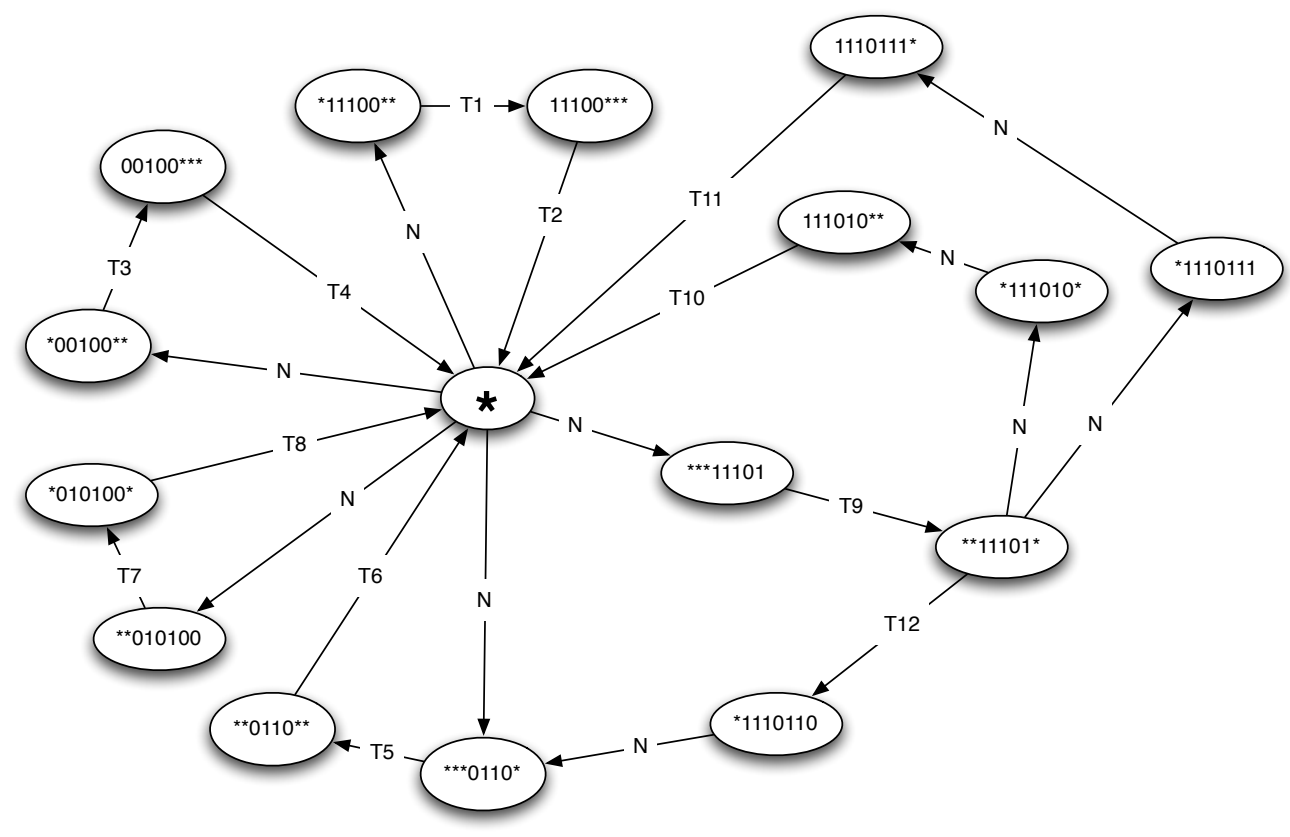

Figure 9: Reduced transitional de Bruijn graph for rule BFO.

$x_{i}$ is even. To show that our rule does indeed have this property, we will use a modification of the de Bruijn graph.

Given a configuration $X=\left(x_{1}, \ldots x_{n}\right)$, one can determine the next iteration, $F(X)$ by reading the edge labels as one traverses the graph from $\left(x_{i-4} \ldots x_{i} \ldots x_{i+3}\right)$ to $\left(x_{i-3} \ldots x_{i} \ldots x_{i+4}\right)$. Since we are considering circular configurations, the traversal of the de Bruijn graph will result in a closed loop. For our purposes, we are not interested in what the actual output is, only if an active transition has occurred. In keeping with that, we are, in fact, only interested in the parts of the graph where such transitions occur. Since a de Bruijn graph for a function of radius 4 can be quite unwieldy, we define a reduced transitional de Bruijn graph modifying the standard de Bruijn graph as follows. First, we label the edges with $T_{i}$ (indicating one of our 12 active state transitions), or $N$, meaning no transition. Second, we draw only those parts of the graph connected to transitions, reducing the rest of the graph to a single node, denoted by an asterisk. Also, where there is no conflict, several nodes leading to or from the same transition are depicted as one, using the notation of the previous section; for example, there is an edge from node $(* 11100 * *)$ to $(11100 * * *)$, because of the various state transitions entailed by $T_{1}$, given all possible values for the $*$ symbols. Furthermore, we ensure that all nodes in the reduced transitional de Bruijn graph are distinct for all values of the $*$ symbol. Finally, for any nodes occurring explicitly in the graph, all adjacent edges are represented, whether they correspond to a transition or not. It is easy to see that parity preservation can be detected from the reduced transitional de Bruijn graph for a given rule; more precisely:

Lemma 12 A rule is parity preserving if and only if any cycle in its reduced transitional de Bruijn graph contains an even number of edges labeled with some transition $T_{i}$.

PROOF Since the output of a circular CA is given by the edge labels of a cycle in its de Bruijn graph, we need only count the transitions to verify parity preservation. Furthermore, the reduced graph compresses only parts of the graphs where no transitions occur. Hence, if the are no cycles containing an odd number 
of transitions in the reduced graph, there can be no configurations leading to an odd number of transitions and vice versa.

By inspecting the transitional de Bruijn graph for rule BFO, and by noticing that there are no cycles containing an odd number of transitions, we then have:

Theorem 5 Rule $\mathrm{BFO}$ is parity preserving.

\subsubsection{Convergence}

We now turn our attention to the more challenging problem of proving that this rule will converge under any conditions. We can think of any CA configuration as an alternating sequence of blocks of 0s and blocks of $1 \mathrm{~s}$ of varying lengths. We will show that BFO eventually converges by showing that its only fixed points are the homogeneous configurations and, furthermore, that any change in the configuration will lead, in a finite number of iterations, to a reduction in the overall number of blocks. Our first lemma shows that every non-homogeneous configuration is changing.

Lemma 13 The only fixed points of rule $\mathrm{BFO}$ are the homogeneous configurations.

PROOF A fixed-point configuration cannot contain pairs of $1 \mathrm{~s}$, since rule pair $\left(T_{5}, T_{6}\right)$ would apply. It cannot contain a block of three or more $1 \mathrm{~s}$ since $\left(T_{1}, T_{2}\right)$ would apply if it is followed by two or more 0 s, and a pair containing $T_{9}$ would apply if it is followed by only one 0 . Therefore, a non-homogeneous fixed-point configuration could only contain isolated $1 \mathrm{~s}$ but the odd length of the configuration would imply that we must have at least two consecutive 0 s, hence the sub-configuration 0100 must occur. If this is preceded by a $0,\left(T_{3}, T_{4}\right)$ apply. If it is preceded by a $01,\left(T_{7}, T_{8}\right)$ apply.

We now wish to show that every transition pair will eventually lead to a reduction in the total number of blocks. We begin with the transition pairs for which this is immediate.

Lemma 14 Transition pairs $\left(T_{5}, T_{6}\right),\left(T_{7}, T_{8}\right)$ and quadruplet $\left(T_{9}, T_{12}, T_{5}, T_{6}\right)$ lead to block reduction in a single step.

Lemma 15 Transition pair $\left(T_{9}, T_{10}\right)$ leads to block reduction in a finite number of steps.

Proof Rule pair $T_{9}: f(* * * 11101 *)=0$ and $T_{10}: f(111010 * * *)=0$ causes the leftward growth of 0 blocks. While a single application of this pair maintains the number of blocks, it leads (possibly through a repeated application of the pair) to an eventual block reduction through either an annihilation or the creation of a single 1:

$$
\begin{aligned}
01111010 \cdots & \leadsto 01101000 \cdots \text { by rules } T_{9} \text { and } T_{10} \\
& \leadsto 00001000 \cdots \text { by rules } T_{5} \text { and } T_{6} \\
0111010 \cdots & \leadsto 0101000 \cdots \text { by rules } T_{9} \text { and } T_{10} \\
& \leadsto 0110000 \cdots \text { by rules } T_{7} \text { and } T_{8} \\
& \leadsto 0000000 \cdots \text { by rules } T_{5} \text { and } T_{6}
\end{aligned}
$$

Notice that $\left(T_{9}, T_{10}\right)$ leads to reducing the number of blocks by either two or four, depending on the parity of the block of $1 \mathrm{~s}$ on which it is acting. Also note that, even though it is possible for the leading block of $1 \mathrm{~s}$ to have shrunk from the left side, while the $\left(T_{9}, T_{10}\right)$ pair is reducing it from the right, one of these two situations will still be reached, since, on the left, the $1 \mathrm{~s}$ can only be eliminated one at a time. 
Lemma 16 Transition pairs $\left(T_{1}, T_{2}\right)$, and $\left(T_{3}, T_{4}\right)$ lead to either reduction or maintenance of the number of blocks.

PROOF Both pairs $T_{1}: f(* 11100 * * *)=1, T_{2}: f(11100 * * * *)=1$, and $T_{3}: f(* 00100 * * *)=1$, $T_{4}: f(00100 * * * *)=1$ are responsible for the rightward growth of 1-blocks. In fact, they grow a block of $1 \mathrm{~s}$ until it either merges with the next block or an isolated 0 preceded by three or more $1 \mathrm{~s}$ is created. At this point, one of the following transition sets will apply: $\left(T_{9}, T_{10}\right)$ if the isolated 0 is followed by 10 , $\left(T_{9}, T_{12}, T_{5}, T_{6}\right)$ if it is followed by 110 , and $\left(T_{9}, T_{11}\right)$ if it is followed by three or more $1 \mathrm{~s}$. We have already seen that the first two cases lead to block reduction, only the latter case can maintain block numbers.

Lemma 17 Transition pair $\left(T_{9}, T_{11}\right)$ leads to reduction or maintenance of the number of blocks in a finite number of steps.

PROOF The pair $T_{9}: f(* * * 11101 *)=0, T_{11}: f(* * * 111011)=0$ is responsible for the start of 0 -growth. This is the only rule pair that initially increases the number of blocks. It is the beginning of the growth of $0 \mathrm{~s}$ from an isolated 0 surround by three or more $1 \mathrm{~s}$ on either side. Once this growth has begun, it is continued by the $\left(T_{9}, T_{10}\right)$ pair until the number of blocks has been returned to its original size or is reduced by two.

Note that in the proof above, if the original number of blocks is maintained, it is because the transitions have produced an isolated 1 with two or more 0s on either side which then begins to grow to the right either merging with the block on the right (and thus reducing the total number of blocks) or creating an isolated 0 which then begins to grow left. What we wish to avoid is a CA which evolves to a periodic configuration of $1 \mathrm{~s}$ growing until only an isolated 0 remains and then shrinking back to an isolated 1 which then regrows. We call this pattern of growing and shrinking the accordion effect. In the next lemma, we show that the accordion effect cannot occur on lattices of odd length.

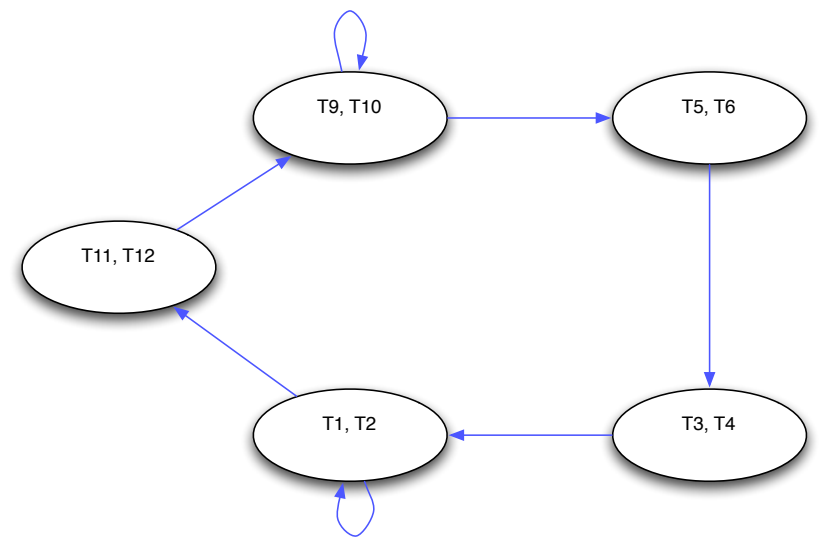

Figure 10: Accordion loop.

Lemma 18 The accordion effect can only occur on lattices of even length.

PROOF Figure 10 shows the transition pairs involved in a cycle of growing and shrinking. If we think of this loop as starting from the $\left(T_{9}, T_{11}\right)$ node, a block of $1 \mathrm{~s}$ is shrinking until the $\left(T_{5}, T_{6}\right)$ node is reached and then beginning with the $\left(T_{3}, T_{4}\right)$ pair, a block of $1 \mathrm{~s}$ begins to grow. Let us assume that the entire lattice is perpetually in some stage of this cycle. We make several observations: 
- The 1-blocks start off having odd length.

- The 0-blocks start off having even length.

- In order for a block of $1 \mathrm{~s}$ to regrow, it must have even length at the end of the shrinking process.

- In order for a 0-block to grow, it must have odd length at the end of the growth of $1 \mathrm{~s}$.

Since the entire configuration is in this process, it is made up of sub-configurations of two forms: $b_{i} w_{i}$, a block of $1 \mathrm{~s}$ followed by a block of 0 s when the $1 \mathrm{~s}$ are growing; or $b_{i} 01 w_{i}$ a block of $1 \mathrm{~s}$ separated by a 01 from a block of $0 \mathrm{~s}$ when the block of $1 \mathrm{~s}$ is shrinking. Consider a block of $0 \mathrm{~s}, w_{1}$ followed by a block of $1 \mathrm{~s}, b_{2}$. If $w_{1}$ has odd length, then it must not change lengths again before the growth of $0 \mathrm{~s}$ restarts otherwise when the block of $1 \mathrm{~s}$ on its left, $b_{1}$ grows, it will merge with $b_{2}$. This means that $\left(T_{9}, T_{11}\right)$ must be applied on the left before $\left(T_{5}, T_{6}\right)$ can be applied on the right. Hence $b_{2}$ will shrink by 1 before the $1 \mathrm{~s}$ have finished shrinking on the right. Since we will need to have an even number of $1 \mathrm{~s}$ at that time, we must now have an odd number of $1 \mathrm{~s}$. In other words, a block of 0 s of odd length is always followed by a block of $1 \mathrm{~s}$ of odd length. Now assume that $b_{1}$ has even length. In this case it must change sizes before the regrowth of $1 \mathrm{~s}$ is complete, so $\left(T_{5}, T_{6}\right)$ must be applies on the right before $\left(T_{9}, T_{11}\right)$ is applied on the left again. Hence $w_{2}$ must already have even length. Taken together, we see that for the accordion effect to endure in perpetuity, the CA must have even length.
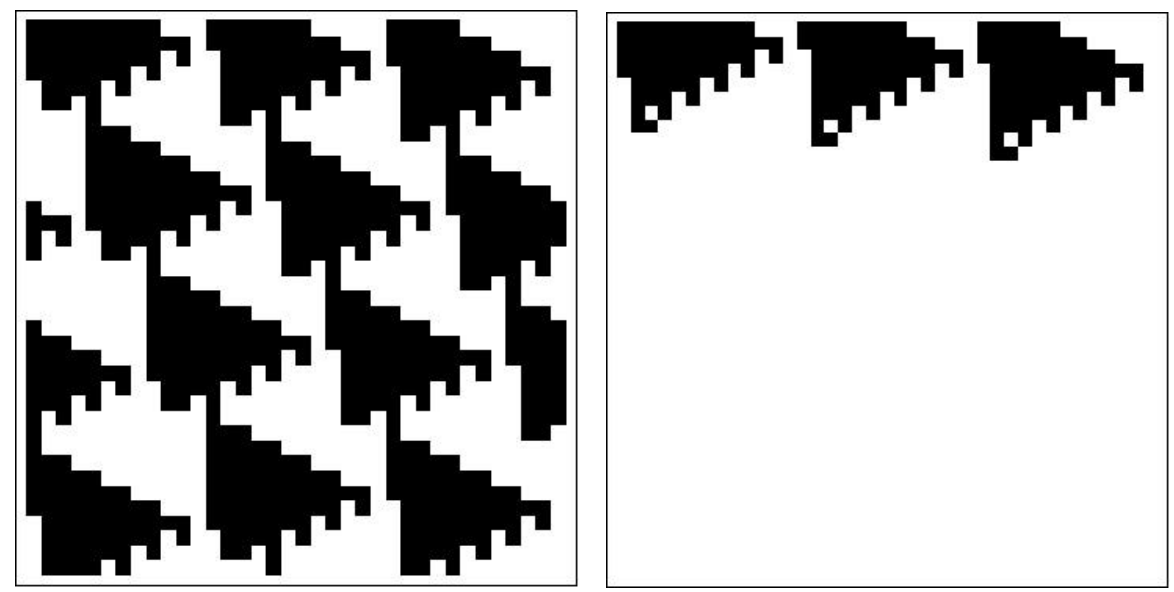

Figure 11: The accordion effect on an even-sized lattice (left) and the successful resolution of an oddsized lattice (right).

Finally, taking these various lemmas together, we have the heart of our convergence proof.

Lemma 19 From any non-homogeneous configuration, the total number of blocks decreases in finite time.

PROOF From the previous lemmas, we see that only rule pair $\left(T_{9}, T_{11}\right)$ increases the number of blocks and that within a finite number of steps, this increase is resolved. Our contention is that the accordion effect is the only way to maintain block numbers. Consider a $\left(T_{9}, T_{11}\right)$ pair occurring anywhere in our CA. If the leading block of $1 \mathrm{~s}$ is odd at the end of the execution of the $\left(T_{9}, T_{10}\right)$ pairs, then the number of blocks had decreased by two. If it is even, the $1 \mathrm{~s}$ will begin to grow right. If the 0-block created by the execution of $\left(T_{9}, T_{11}\right)$ is unchanged from the right, then it has even length and the regrowth of $1 \mathrm{~s}$ will 
result in a merger of $1 \mathrm{~s}$ and a reduction in the number of blocks. Now the only way for the 0-block to have changed on the right is if the 1-block on its right had shrunk due to the application of $\left(T_{9}, T_{10}\right)$ or $\left(T_{9}, T_{11}\right)$. If the reduction in the 1-block was not initiated by the $\left(T_{9}, T_{11}\right)$ pair, then real reduction in the number of blocks has occurred. If it was initiated by $\left(T_{9}, T_{11}\right)$, then block reduction can only be prevented if another $\left(T_{9}, T_{11}\right)$ pair is being executed to its right. Arguing in this way, we see that reduction in total block number can only be avoided if the $\mathrm{CA}$ is experiencing the accordion effect which can only occur in even-sized lattices.

From Theorem 5 and Lemmas 13 and 19 , we obtain:

Theorem 6 Given a CA of odd length, rule BFO converges to all 1s if the initial configuration has odd parity and to all 0 s if it has even parity.

\section{Concluding Remarks}

In this paper, we have established upper and lower bounds on the radius of rules that solve the parity problem by showing that there exists a rule of radius 4 which converges to all $1 \mathrm{~s}$ if the initial configuration is odd, and to all $0 \mathrm{~s}$ if it is even and, further, by proving that this problem is unsolvable by rules of radius 2 , even with the less strict condition of prime-sized lattices. The corresponding questions for radius 3 remain open. However, we have developed tools in this paper that should be helpful in solving this latter problem as well.

It is clear by now, how painstaking the task of designing a CA rule can be, let alone the formal proof of its correctness. To some extent, the process reminds us of similar programming efforts on simple, pre-modern computational models, such as Turing machines. And in this sense, we are still indeed at this point in history, when programming cellular automata.

Since our main motivation for addressing the parity problem is not conscribed to it, one may ask how generalisable our experience herein could be to related problems, including the parity problem for radius 3 , as well as other related computational problems for CAs. It is tempting to think of the possibility of implementing a high-level programming approach that would automatically generate the state transitions of a CA rule, given the kinds of notions we have used, such as the growth of blocks of a given size in a given direction, the annihilation of blocks of given kind, etc. Even if this form of programming, so to speak, by patterns, does not solve the problem of designing a rule (the target algorithm), at least it would help its high-level conception, and its implementation in terms of the required state transitions.

As a methodological note, it is worth mentioning that it was demanding in practice to resort to computational aids to complement the formal efforts. After all, the details involved in rule design are so many that it is quite easy to overlook some of them. This turned out to be essential in the present case for fine tuning our design in its origin. Such an interplay between formal and computational methods also came into play for devising the most compact representation of the BFO rule, as shown in the paper, for enumerating all cycles of length 7 in the de Bruijn graph of radius 2, and for the evaluation of the radius 2 rules that had retained potential for being perfect solvers of the parity problem, by not violating the constraints derived in the proof, at their various stages of development.

\section{Acknowledgements}

This work has been partially supported by the Natural Sciences and Engineering Research Council of Canada (NSERC), by Prof. Flocchini's University Research Chair, and by a grant provided to P.P.B.O., 
by MackPesquisa - Fundo Mackenzie de Pesquisa.

\section{References}

[1] D. Angluin (1980): Local and Global Properties in Networks of Processors. In: Proceedings of the 28th Symposium on Theoretical Aspects of Computer Science (STACS), pp. 82-93, doi: $10.1145 / 800141.804655$.

[2] D. Angluin, J. Aspnes, D. Eisenstat \& E. Ruppert: (2007): The computational power of population protocols. Distributed Computing 20(4), pp. 279-304, doi:10.1007/s00446-007-0040-2.

[3] P.P. Chaudhuri, D.R. Chowdhury, S. Nandi S \& S. Chattopadhyay (1997): Additive cellular automata: Theory and applications. 1, IEEE Computer Society Press: Los Alamitos.

[4] N. Fatès (2011): Stochastic Cellular Automata Solve the Density Classification Problem with an Arbitrary Precision. In: Proceedings of the 28th Symposium on Theoretical Aspects of Computer Science (STACS), pp. 284-295, doi:10.4230/LIPIcs.STACS.2011.284.

[5] H. Fukś (1997): Solution of the density classification problem with two cellular automata rules. Physical Review E 55(3), pp. R2081-R2084, doi:10.1103/PhysRevE.55.R2081

[6] D. Griffeath \& C. Moore, editors (2003): New constructions in cellular automata. Oxford Univ. Press - Santa Fe Institute Studies on the Sciences of Complexity.

[7] S.O. Haykin (2008): Neural networks and learning machines, 3rd edition. Prentice Hall.

[8] J.E. Hopcroft, R. Motwani \& J.D. Ullman (2006): Introduction to Automata Theory, Languages, and Computation, 3rd edition. Addison-Wesley.

[9] K.M. Lee, H. Xu \& H.F. Chau (2001): Parity problem with a cellular automaton solution. Physical Review E 64(2), pp. 026702/1-026702/4, doi:10.1103/PhysRevE.64.026702.

[10] C. Lenzen, Y.A. Oswald \& R. Wattenhofer (2008): What can be approximated locally?: case study: dominating sets in planar graphs. In: Proc. 20th Annual ACM Symposium on Parallelism in Algorithms and Architectures (SPAA), pp. 46-54, doi:10.1145/1378533.1378540

[11] C.L.M. Martins \& P.P.B. de Oliveira (2009): Improvement of a result on sequencing elementary cellular automata rules for solving the parity problem. Electronic Notes in Theoretical Computer Science 252, pp. 103-119, doi:10.1016/j.entcs.2009.09.017.

[12] P.P.B. de Oliveira, J.C. Bortot \& G.M.B. Oliveira (2006): The best currently known class of dynamically equivalent cellular automata rules for density classification. Neurocomputing 70(1-3), pp. 35-43, doi $10.1016 /$ j.neucom.2006.07.003.

[13] D. Peleg (2000): Distributed Computing: A Locality-Sensitive Approach. SIAM Monographs on Discrete Mathematics and Applications.

[14] M. Sipper (1998): Computing with cellular automata: Three cases for nonuniformity. Physical Review E 57(3), pp. 3589-3592, doi 10.1103/PhysRevE.57.3589.

[15] B. Voorhees (2009): Additive cellular automata. In R.A. Meyers, editor: Encyclopedia of Complexity and Systems Science, Elsevier, pp. Entry 101, 17 pages, doi 10.1007/978-0-387-30440-3_4

[16] D. Wolz \& P.P.B. de Oliveira (2008): Very effective evolutionary techniques for searching cellular automata rule spaces. Journal of Cellular Automata 3(4), pp. 289-312. 